\title{
TERAPEUTAS ALTERNATIVOS EN MÉXICO Y LA ESTRATEGIA DE LA OMS SOBRE MEDICINA TRADICIONAL 2014-2023: COMUNICACIÓN, CREENCIAS Y FACTORES SOCIO-ECONÓMICOS
}

\author{
Dra. Mavi Corell-Doménech \\ Florida Universitària, Catarroja, España \\ mcorell@florida-uni.es \\ ORCID iD: https://orcid.org/0000-0002-3785-3897
}

Recibido el 19 de agosto de 2018

Aceptado el 26 de noviembre de 2018

\section{Resumen}

Esta investigación constituye un estudio descriptivo de corte cualitativo que analiza los testimonios de 12 terapeutas especialistas en medicinas o terapias complementarias de Ciudad de México y el Estado de México con el fin de valorar si sus prácticas responden a las recomendaciones de la Estrategia de la OMS sobre medicina tradicional 2014-2023 de la Organización Mundial de la Salud. Esta organización promueve la medicina tradicional y complementaria por razones socio-económicas y culturales en países como México por su contribución al bienestar y a una atención a la salud centrada en las personas.

Entre los resultados obtenidos observamos que los terapeutas entrevistados muestran capacidad de observación y empatía con sus pacientes, consideran decisivos los estados emocionales en los procesos de salud-enfermedad y sus honorarios son flexibles. En algunos casos plantean la utilización de las terapias complementarias en enfermedades graves como el cáncer, lo que suscita un debate ético y constata que, como indica la OMS en el citado documento, es necesario promover un uso racional de estas prácticas. Concluimos que la incorporación de productos, profesionales y prácticas al sistema sanitario mexicano requiere todavía continuar investigando y reglamentando la práctica, formación y productos con el fin de garantizar su eficacia y la seguridad de los usuarios.

Palabras clave: Terapias Alternativas Y Complementarias, Medicina Tradicional, Organización Mundial De La Salud, Medicina Doméstica, Educación Para La Salud. 


\title{
ALTERNATIVE THERAPISTS IN MEXICO AND THE WHO TRADITIONAL MEDICINE STRATEGY 2014- 2023 OF THE WORLD HEALTH ORGANIZATION: COMUNICATION, BELIEFS AND SOCIO-ECONOMIC FACTORS
}

\begin{abstract}
This research constitutes a qualitative study that analyses the declarations of 12 alternative therapists from urban environments in both Mexico City and the State of Mexico in order to assess whether their practices respond to the recommendations of the WHO Traditional Medicine Strategy 2014 - 2023. The World Health Organization (WHO) promotes the introduction of these practices for socio-economic and cultural reasons in Mexico. The declarations confirm that alternative and complementary therapies can contribute to wellbeing and people-centered health care.
\end{abstract}

Among the results obtained, we observed that the interviewed therapists demonstrated capacity of observation, showed empathy with their patients, considered the emotional states of their patients as being decisive in health-disease processes, and also that their fees were flexible. In some cases, therapists propose the use of complementary therapies in serious diseases such as cancer, which provokes an ethical debate and confirms that it is necessary to promote the rational use of these practices as indicated by the WHO in the aforementioned document. We conclude that the incorporation of products, professionals and practices to the Mexican health system requires continued investigation and regulation of the practice, training and products in order to guarantee their effectiveness and the safety of users.

Keywords: Alternative and Complementary Therapies, Traditional Medicine, World Health Organization, Domestic Medicine, Health Education.

\section{Cómo citar este artículo:}

Corell-Doménech, M. (2019). "Terapeutas alternativos en México y la Estrategia de la OMS sobre medicina tradicional 2014-2023 de la Organización Mundial de la Salud: comunicación, creencias y factores socio-económicos", en Perspectivas de la Comunicación, Vol. 12, nº 1. pp. 59-77.

Agradecimientos: El presente trabajo ha sido desarrollado en el marco del proyecto de investigación "Estudio y clasificación de las terapias naturales, complementarias y alternativas a través de los medios de comunicación y de las redes sociales. Ideas y valores de transferencia al imaginario social" (ESTENAS) (CSO2014-57778-R), financiado por el Ministerio de Economía y Competitividad y por fondos FEDER de la Unión Europea. La IP del proyecto es Carolina Moreno-Castro (Universitat de València). 
Introducción

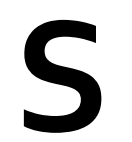

iguiendo las recomendaciones de la Organización Mundial de la Salud (OMS), la Secretaría de Salud del gobierno mexicano (SSGM) está trabajando para que, junto a las medicinas tradicionales, las terapias o medicinas complementarias formen parte de los servicios de salud (SSGM, 2018a y 2018b). En el mismo sentido apunta la Resolución Ao/2009/14 del Parlamento Latinoamericano que aprueba la Ley Marco en Materia de Medicinas Complementarias para América Latina y el Caribe, para regular la práctica, enseñanza e investigación de las medicinas complementarias que posteriormente cada país debe adaptar a sus circunstancias concretas. Este marco legal recomienda incorporar a los Sistemas Nacionales de Salud la acupuntura, la homeopatía, la quiropráctica y la herbolaria medicinal ( $\mathrm{PL}, 2009)$.

En el presente trabajo se analiza el discurso de una muestra de terapeutas complementarios de las zonas urbanas de Ciudad de México y del Estado de México en relación a las recomendaciones de la Estrategia de la OMS sobre medicina tradicional 20142023 (OMS, 2013). Esta institución, que agrupa la medicina tradicional junto a la complementaria bajo el acrónimo MTC, define la medicina tradicional como aquellos "conocimientos, capacidades y prácticas basados en las teorías, creencias y experiencias propias de diferentes culturas, bien sean explicables o no, utilizadas para mantener la salud y prevenir, diagnosticar, mejorar o tratar enfermedades físicas y mentales" y la medicina complementaria la que se refiere a "un amplio conjunto de prácticas de atención de salud que no forman parte de la tradición ni de la medicina convencional de un país dado ni están totalmente integradas en el sistema de salud predominante (OMS, 2013, p.15).

En su estrategia, la OMS recomienda aprovechar "la contribución potencial de la MTC a la salud, el bienestar y la atención de salud centrada en las personas", promover "la utilización segura y eficaz de la MTC a través de la reglamentación y la investigación", e incorporar "productos, profesionales y prácticas en los sistemas de salud" (OMS, 2013, p. 11). Asimismo, hay que destacar que la OMS plantea profundizar en las recomendaciones referentes al periodo 2002-2005 que proponían "promover la seguridad, eficacia y calidad de la medicina tradicional mediante la ampliación de la base de conocimientos y la prestación de asesoramiento sobre normas reglamentarias y de garantía de la calidad" y "el uso terapéutico racional de la medicina tradicional entre los profesionales y los usuarios" (OMS, 2013, p. 11).

En cuanto a las especialidades contempladas, la OMS afirma que "las prácticas de MTC son muy distintas de un país a otro, y algunas (a veces llamadas modalidades) se consideran de diferentes maneras en función de la cultura, el conocimiento y la accesibilidad de la medicina convencional" (OMS, 2013, p. 31). Asimismo, considera que "las prácticas de MTC incluyen medicamentos terapéuticos y tratamientos de salud basados en procedimientos, por ejemplo a base de hierbas, naturopatía, acupuntura y terapias manuales tales como la quiropráctica, la osteopatía y otras técnicas afines, incluidos qi gong, tai chi, yoga, medicina termal y otras terapias físicas, mentales, espirituales y psicofísicas" (OMS, 2013: 31). En cuanto a los terapeutas, denominados por la OMS "profesionales de MTC", pueden ser prácticos de medicina tradicional o de medicina complementaria, médicos convencionales $\mathrm{u}$ otros profesionales sanitarios (OMS, 2013, p. 29). 
Los recursos y la atención sanitaria que elige un usuario dependen de su contexto económico, social y cultural, así como de las representaciones sociales de los procesos de salud-enfermedad (Menéndez, 1994 y 2002; Pasarín, 2011). De esta manera los individuos desarrollan diferentes estrategias para solucionar su problema de salud (Haro, 2000; Idoyaga Molina, 2002). El factor económico es determinante (OMS, 2013: 29). Trabajos previos demuestran que, en México, un número creciente de usuarios incluye a las terapias complementarias en su itinerario terapéutico por su menor coste en relación a la medicina convencional (Molina-Rodríguez, 2006), uso de medicamentos e ingresos hospitalarios (Kooreman et al., 2012).

La conjunción de una serie de factores determinó que la esperanza de vida de los mexicanos fuera de 75 años en 2015, una de las más bajas de la Organización para la Cooperación y el Desarrollo Económicos (OCDE) (OCDE, 2017). El 43,6\% de la población mexicana vive en condiciones de pobreza, una lacra que afecta al 77,6\% de los indígenas de este territorio (OMS, 2018). Entre las principales causas de mortalidad de los mayores de 40 años figura la obesidad y el sobrepeso, y en relación a ellas son prevalentes las enfermedades crónicas no trasmisibles (diabetes, enfermedades cardiacas, cerebrovasculares, cirrosis y cáncer). Los mexicanos tienen la segunda prevalencia más alta de obesidad (33\% de los adultos), y la más alta proporción general de sobrepeso u obesidad (73\%) de la OCDE (OCDE, 2017).

Hay que destacar que la población mexicana comparte el sistema de creencias de las terapias complementarias y se muestra escéptica hacia los logros de la ciencia y la tecnología. Según la última encuesta del Consejo Nacional de Ciencia y Tecnología (CONACYT), el 74,56\% de los encuestados está muy de acuerdo o de acuerdo en que las enfermedades pueden tratarse adecuadamente por medios no reconocidos por la ciencia, como son las mencionadas terapias (CONACYT, 2016). Asimismo, el estudio de TorresVaca (2007) sobre la percepción de las terapias complementarias de estudiantes mexicanos universitarios muestra que el $73 \%$ las acepta, bien en exclusiva o en un servicio combinado en las unidades de atención primaria del sector salud, y el $72 \%$ tenía un consultorio de medicinas tradicionales y terapias alternativas en su colonia.

Distintas instituciones mexicanas han iniciado el proceso de regulación de las terapias complementarias siguiendo las recomendaciones de la OMS. A continuación exponemos algunos de estos marcos legales. Entre las terapias reguladas total o parcialmente por el Sistema de Salud mexicano figura la herbolaria, relacionada con las tradiciones indígenas y el oficio de herbero/a que, como parte de la medicina tradicional, goza de gran popularidad en México. En cuanto a la herbolaria están proliferando las empresas que comercializan suplementos nutricionales, vitaminas y probióticos herbales y no herbales que constituyen un mercado en expansión. La Comisión Federal para la Protección contra Riesgos Sanitarios (COFEPRIS) ha regularizado compuestos de hierbas, extractos vegetales, alimentos tradicionales, deshidratados o concentrados de frutas, con o sin vitaminas o minerales añadidos que se pueden presentar en forma farmacéutica (COFEPRIS, 2016a y 2016b).

Aunque la acupuntura proviene del sistema médico chino, goza también de gran aceptación en México y está regulada en su enseñanza y práctica. La Dirección General de Calidad y Educación en Salud ha establecido unos criterios para guiar la evaluación de los planes y programas de la formación del técnico superior universitario en acupuntura. La ley prohíbe que se emplee la acupuntura como tratamiento único de "malformaciones 
congénitas y adquiridas, tumores benignos y malignos, infecciones bacterianas graves, infecciones virales; $\mathrm{VIH}$, hepatitis y padecimientos que impliquen cirugía mayor", y en aquellos casos restringidos por otras Normas Oficiales Mexicanas, como personas con sobrepeso y obesidad, salvo "como paliativo del dolor y terapéutica complementaria, para brindar una mejor calidad de vida de los pacientes con enfermedades en etapa terminal" (SSGM, 2012, p. 6).

Por su parte la quiropraxia, que en la medicina tradicional encuentra su equivalente en la figura del huesero/a, tiene la ventaja de su bajo coste para tratar trastornos neuromusculoesqueléticos, según la OMS. México cuenta ya con las guías de estructuración y de evaluación de planes y programas para la formación del licenciado en quiropráctica, elaboradas por la Comisión Interinstitucional para la Formación de Recursos Humanos en Salud, junto a la homeopatía, la acupuntura y la herbolaria (CIFRHS, 2015).

Finalmente, la homeopatía, terapia que difundieron homeópatas españoles en el México rural de mediados del siglo XIX (Hernández, 2017), puede cursarse como especialidad en la Escuela Nacional de Medicina y Homeopatía del Instituto Politécnico Nacional, dependiente de la Secretaría de Educación Pública, y en la Escuela Libre de Homeopatía, de carácter privado. Si bien esta terapia se encuentra institucionalizada, como muestra la financiación con dinero público del Hospital Nacional Homeopático de Ciudad de México, uno de los más grandes de América latina, la existencia de este centro es cuestionada por un número no despreciable de médicos y científicos mexicanos.

\section{Material y método}

Esta investigación constituye un estudio descriptivo de corte cualitativo. A continuación se exponen las técnicas empleadas para la búsqueda y registro de la información, así como para el análisis y discusión de los resultados obtenidos.

\section{Fases del estudio}

El trabajo de campo se desarrolló en tres etapas de diciembre de 2016 a septiembre de 2017. En una primera fase se realizó una exploración con el objetivo de obtener información para diseñar el cuestionario de la entrevista semiestructurada mediante la técnica de investigación social (Alonso, 1995). Se eligió como forma de registro la entrevista con el fin de preservar y dar valor al testimonio de los terapeutas y siguiendo los estudios de historia oral de Crano et al. (2014), Denscombe (2014), Marshall y Rossman (2014) y Yow (2014). El cuestionario fue validado por cuatro miembros del grupo de investigación ESTENAS que incorporaron las sugerencias y los problemas que habían tenido en las entrevistas previas realizadas con estas mismas preguntas durante un trabajo de campo realizado en 2016.

En una segunda fase se realizaron persona a persona las entrevistas, se registraron en audio de junio a agosto de 2017 y se transcribieron las grabaciones, eliminando ruidos, cacofonías y anacolutos de los hablantes. En una tercera fase, tras varias lecturas de las transcripciones, se analizaron las respuestas de los entrevistados y entrevistadas para valorar si respondían a las recomendaciones de la estrategia sobre medicina tradicional y complementaria de la OMS.

\section{Muestra}


Se eligieron especialistas en las terapias más destacadas contempladas por la Secretaría de Salud (SSGM, 2018a) y el marco teórico del trabajo residentes en zonas urbanas de Ciudad de México y del Estado de México. Dos homeópatas, tres terapeutas de medicina natural, dos acupuntores y tres terapeutas de especialidades relacionadas con manipulaciones del cuerpo (un quiropráctico, una osteópata y un hidroterapeuta). Asimismo, se ha incluido en la muestra dos terapeutas que se denominan a sí mismos biomagnetoterapeuta y médico cuántico, respectivamente, pero que aplican, además, terapias contempladas por la Secretaría de Salud: homeopatía, medicina herbal y medicina natural.

Una vez se contactó con los terapeutas, se explicaron los objetivos de la entrevista y la confidencialidad de las declaraciones. Las entrevistas transcurrieron en sus consultorios privados y en la Universidad de Chapingo, centro que imparte cursos en terapias complementarias. El consentimiento informado de cada uno de los participantes se obtuvo de manera escrita. Se ha utilizado un nombre ficticio para preservar el anonimato de los participantes, cuyas características sociodemográficas se muestran en la Tabla I, y la denominación que ellos utilizaron para designarse a sí mismos según su especialidad.

Las características sociodemográficas de los entrevistados incluyen su edad, lugar de residencia, estudios y lugar de estudios, ocupación actual y las terapias que practican que, como se ha dicho, con la excepción de los médicos homeópatas, son más de una. Este trabajo no expone los mecanismos curativos de las terapias complementarias, que carecen en todos los casos del aval de la medicina científica, una cuestión que, por otra parte, la Organización Mundial de la Salud tampoco analiza en su estrategia sobre MTC.

\begin{tabular}{|c|c|c|c|c|c|c|}
\hline Nombre & Edad & $\begin{array}{l}\text { Lugar de } \\
\text { residencia }\end{array}$ & Estudios & Centro de estudios & Ocupación & $\begin{array}{l}\text { Terapias que } \\
\text { practica }\end{array}$ \\
\hline Martín & 28 & $\begin{array}{l}\text { Estado de } \\
\text { México }\end{array}$ & $\begin{array}{l}\text { Médico } \\
\text { cirujano } \\
\text { homeópata }\end{array}$ & $\begin{array}{l}\text { Escuela Nacional } \\
\text { de Medicina y } \\
\text { Homeopatía del } \\
\text { Instituto } \\
\text { Politécnico } \\
\text { Nacional }\end{array}$ & $\begin{array}{l}\text { Médico de la } \\
\text { Secretaría de Salud de } \\
\text { México }\end{array}$ & $\begin{array}{l}\text { Medicina } \\
\text { convencional, } \\
\text { homeopatía }\end{array}$ \\
\hline Gong & 38 & $\begin{array}{l}\text { Ciudad de } \\
\text { México }\end{array}$ & $\begin{array}{l}\text { Médico, } \\
\text { especialista en } \\
\text { medicina } \\
\text { china }\end{array}$ & $\begin{array}{l}\text { Universidad en } \\
\text { China }\end{array}$ & $\begin{array}{l}\text { Acupuntor. Trabaja } \\
\text { por cuenta propia }\end{array}$ & $\begin{array}{l}\text { Acupuntura, } \\
\text { masaje, } \\
\text { herbolaria }\end{array}$ \\
\hline Felisa & 38 & $\begin{array}{l}\text { Ciudad de } \\
\text { México }\end{array}$ & $\begin{array}{l}\text { No médico, } \\
\text { especialista en } \\
\text { acupuntura }\end{array}$ & $\begin{array}{l}\text { Universidad de } \\
\text { Chapingo }\end{array}$ & $\begin{array}{l}\text { Acupuntora. Trabaja } \\
\text { por cuenta propia }\end{array}$ & $\begin{array}{l}\text { Acupuntura, } \\
\text { masaje, } \\
\text { herbolaria }\end{array}$ \\
\hline Lázaro & 38 & $\begin{array}{l}\text { Ciudad de } \\
\text { México }\end{array}$ & $\begin{array}{l}\text { Licenciado en } \\
\text { fisioterapia }\end{array}$ & $\begin{array}{l}\text { Escuela Mexicana } \\
\text { de Fisioterapia }\end{array}$ & $\begin{array}{l}\text { Hidroterapeuta. } \\
\text { Trabaja por cuenta } \\
\text { propia }\end{array}$ & $\begin{array}{l}\text { Masaje, } \\
\text { hidroterapia }\end{array}$ \\
\hline Ramón & 52 & $\begin{array}{l}\text { Ciudad de } \\
\text { México }\end{array}$ & $\begin{array}{l}\text { Licenciado en } \\
\text { educación } \\
\text { física }\end{array}$ & $\begin{array}{l}\text { Universidad de } \\
\text { Chapingo }\end{array}$ & $\begin{array}{l}\text { Quirofísico. Trabaja } \\
\text { por cuenta propia }\end{array}$ & $\begin{array}{l}\text { Quiropraxia, } \\
\text { masaje, } \\
\text { masaje para } \\
\text { deportistas y } \\
\text { acróbatas }\end{array}$ \\
\hline Berenice & & $\begin{array}{l}\text { Ciudad de } \\
\text { México }\end{array}$ & $\begin{array}{l}\text { Licenciada en } \\
\text { fisioterapia }\end{array}$ & $\begin{array}{l}\text { Hospital Inglés de } \\
\text { México }\end{array}$ & $\begin{array}{ll}\text { Osteópata } & \text { y } \\
\text { administradora en la }\end{array}$ & Osteopatía \\
\hline
\end{tabular}




\begin{tabular}{|l|l|l|l|l|l|l|}
\hline & & & & & $\begin{array}{l}\text { Escuela de Osteopatía } \\
\text { de Madrid en México }\end{array}$ & \\
\hline Juan & 68 & $\begin{array}{l}\text { Ciudad de } \\
\text { México }\end{array}$ & $\begin{array}{l}\text { Médico } \\
\text { cirujano } \\
\text { homeópata }\end{array}$ & $\begin{array}{l}\text { Facultad } \\
\text { Medicina de la } \\
\text { UNAM }\end{array}$ & $\begin{array}{l}\text { Homeópata. Trabaja } \\
\text { por cuenta propia }\end{array}$ & Homeopatía \\
\hline
\end{tabular}




\begin{tabular}{|c|c|c|c|c|c|c|}
\hline Abraham & & $\begin{array}{l}\text { Ciudad } \\
\text { de } \\
\text { México }\end{array}$ & $\begin{array}{l}\text { Médico cirujano, } \\
\text { cursos de } \\
\text { ozonoterapia, } \\
\text { medicina cuántica, } \\
\text { entre otros }\end{array}$ & $\begin{array}{ll}\text { Facultad de } \\
\text { Medicina de } \\
\text { la UNAM }\end{array}$ & $\begin{array}{l}\text { Médico especialista en } \\
\text { medicina cuántica. Trabaja } \\
\text { por cuenta propia }\end{array}$ & $\begin{array}{l}\text { Medicina } \\
\text { cuántica, } \\
\text { medicina } \\
\text { natural, } \\
\text { ozonoterapia, } \\
\text { herbolaria, } \\
\text { iridiología } \\
\end{array}$ \\
\hline Daniel & 58 & $\begin{array}{l}\text { Ciudad } \\
\text { de } \\
\text { México }\end{array}$ & $\begin{array}{lr}\text { Médico cirujano, } \\
\text { postgrado } \\
\text { acupuntura, cursos } \\
\text { de medicina natural, } \\
\text { entre otros }\end{array}$ & $\begin{array}{l}\text { Facultad de } \\
\text { Medicina de } \\
\text { la UNAM, }\end{array}$ & $\begin{array}{l}\text { Médico naturista. Trabaja } \\
\text { por cuenta propia y es } \\
\text { formador en la Universidad } \\
\text { de Chapingo }\end{array}$ & $\begin{array}{l}\text { Medicina } \\
\text { natural, } \\
\text { acupuntura, } \\
\text { herbolaria, } \\
\text { iridiología, } \\
\text { biomagneto- } \\
\text { terapia, } \\
\text { cromoterapia }\end{array}$ \\
\hline José Luis & 58 & $\begin{array}{l}\text { Ciudad } \\
\text { de } \\
\text { México }\end{array}$ & $\begin{array}{l}\text { Ingeniero químico } \\
\text { industrial, Posgrado } \\
\text { de Etnobotánica } \\
\text { médica }\end{array}$ & $\begin{array}{l}\text { Instituto } \\
\text { Politécnico } \\
\text { Nacional } \\
\text { (IPN) }\end{array}$ & $\begin{array}{l}\text { Médico naturista. Trabaja } \\
\text { por cuenta propia }\end{array}$ & $\begin{array}{l}\text { Medicina } \\
\text { natural, } \\
\text { herbolaria, } \\
\text { masaje }\end{array}$ \\
\hline Marisa & 65 & $\begin{array}{l}\text { Ciudad } \\
\text { de } \\
\text { México }\end{array}$ & $\begin{array}{l}\text { Licenciada en } \\
\text { pedagogía, cursos de } \\
\text { nutrición, } \\
\text { biomagnetismo, } \\
\text { herbolaria, entre } \\
\text { otros }\end{array}$ & & $\begin{array}{l}\text { Biomagnetoterapeuta. } \\
\text { Trabaja por cuenta propia }\end{array}$ & $\begin{array}{l}\text { Biomagneto- } \\
\text { terapia, } \\
\text { medicina } \\
\text { natural, } \\
\text { iridiología }\end{array}$ \\
\hline Anabel & 39 & $\begin{array}{l}\text { Estado } \\
\text { de } \\
\text { México }\end{array}$ & $\begin{array}{lr}\text { Terapeuta } & \text { de } \\
\text { medicina Licenciada } \\
\text { en Biología y } \\
\text { postgrado } \\
\text { Botánica } \\
\text { natural } \\
\text { complementaria }\end{array}$ & $\begin{array}{l}\text { UNAM } \\
\text { Universidad } \\
\text { de Chapingo }\end{array}$ & $\begin{array}{l}\text { Terapeuta de medicina } \\
\text { natural. Trabaja por cuenta } \\
\text { propia y es formadora en la } \\
\text { Universidad de Chapingo }\end{array}$ & $\begin{array}{l}\text { Herbolaria, } \\
\text { medicina } \\
\text { energética, } \\
\text { medicina } \\
\text { natural }\end{array}$ \\
\hline
\end{tabular}

Tabla I. Características sociodemográficas de los terapeutas complementarios de México entrevistados

\section{Resultados}

A continuación se ofrecen los testimonios de los y las terapeutas entrevistados en relación a las recomendaciones de la estrategia sobre medicina tradicional y complementaria de la OMS. En el apartado de conclusiones se analizan y discuten estos resultados.

\section{- Descripción de los servicios del terapeuta}

Los terapeutas entrevistados ofrecen sus servicios en su consultorio privado, trabajan en solitario y, en dos de los casos, con su pareja. Solo el médico cuántico tiene un ayudante. En la Universidad de Chapingo, durante las formaciones, ofrecen servicios gratuitos o de bajo coste. 
Todos los entrevistados afirman que sus pacientes observan mejorías en su estado de salud a partir de la primera sesión de terapia, que dura entre 60 y 90 minutos. Las siguientes sesiones son de 30 minutos como mínimo en todos los casos. El número de sesiones necesarias para que el paciente obtenga los efectos deseados oscila de dos a tres, con la excepción de las terapias manuales - quiropraxia e hidroterapia- y la medicina cuántica que requieren entre cuatro y seis.

Diez de los terapeutas entrevistados cobran de acuerdo con las posibilidades económicas del paciente: de o a 500 pesos ( 25 euros), excepto uno de los homeópatas que cobra de o a 1.000 pesos. La primera visita de la osteópata tiene un coste de 900 pesos y la del médico cuántico, 1.500 pesos. Los equipos que utilizan para sus tratamientos no son costosos; por ejemplo, el necesario para aplicar el biomagnetismo implica una inversión de 28.000 pesos (1.400 euros). Con la excepción de los médicos homeópatas que únicamente practican la homeopatía en un caso y la homeopatía y la medicina convencional en el otro, el resto son especialistas en una o dos terapias y practican varias.

"El porcentaje más alto de la población es de gente de bajos recursos; uno menos alto es de gente de clase media y el más bajo es de la clase alta, de gente que tiene acceso a ciertas cosas", explica Berenice, osteópata. Añade que la población indígena de las zonas rurales prefiere acudir a las terapias complementarias por su relación con la medicina tradicional: "Dentro de la clase baja hay muchos pueblos o zonas donde todavía hay indígenas que trabajan cosas naturales, cosas alternativas. Pero toda esta área, en México, no está regulada. Hay mucha gente que, en lugar de ir al centro de salud, prefiere ir con el huesero de la zona o del pueblo, o van donde el hierbero de la zona. Y eso hace muy complicada una regulación, porque hay muchos estados que tienen pueblos muy pobres, que no tienen el acceso necesario como para ir al centro de salud", añade.

Ocho entrevistados coinciden en que la mayor parte de sus pacientes llegan a ellos por tres dolencias: crónicas, estrés o ansiedad u otras patologías derivadas de la forma de vida actual o porque se han tratado mediante la medicina convencional sin éxito. Berenice, osteópata, lo explica así:

"Generalmente es un paciente crónico que ya ha ido a la medicina convencional, que ha buscado de diferentes maneras una respuesta médica y no ha encontrado algo específico. Somos los últimos de los últimos a dónde llegan. Esa es la realidad que sucede actualmente".

Durante la primera sesión todos los terapeutas realizan una historia clínica exhaustiva a partir de la observación, exploración física del paciente y entrevista, mediante técnicas variadas, como explica Daniel, médico naturista: "En una primera sesión evaluamos las condiciones del paciente en formato de historia clínica. Yo soy médico y tengo una formación que no rechazo en ese sentido y que me permite ordenar los datos de mi paciente de forma sistemática. Entonces evalúo a mi paciente con estudios de laboratorio o técnicas alternativas de validación: diagnóstico iridiológico, lingual, pulsátil, que son técnicas chinas. Después del diagnóstico pasamos a la terapéutica: acupuntura, biomagnetismo, cromoterapia. Le hago un programa de vida sana. Modificaciones en los hábitos de alimentación y recursos de aplicación doméstica de la medicina natural. Hago una prescripción de medicamento. Intento no utilizar la farmacia convencional. Encuentro mucho beneficio en la medicina herbolaria". 
A continuación establecen un diagnóstico y aplican la terapia más adecuada. Todos los entrevistados mencionan en algún momento de la entrevista la alimentación inadecuada como uno de los aspectos fundamentales a mejorar por parte de sus pacientes. Plantean al paciente un programa de vida sana que debe desarrollar en el entorno doméstico y que incluye dieta y ejercicio físico. "Estructuramos modificaciones de los hábitos de alimentación con una serie de recursos de aplicación doméstica de la medicina natural", explica Daniel, médico naturista. "Lo que nos cuesta es que la gente modifique su esquema de vida. Nadie quiere comer bien, hacer ejercicio o higienizarse transitoriamente, a menos que lleguen a una enfermedad grave. Son los pacientes más enfermos los que están dispuestos a cambiar y te muestran el verdadero alcance de un procedimiento no convencional", añade.

Juan, médico homeópata, considera que la curación está en manos del paciente: "En una consulta homeopática, donde hablamos para conocernos, queremos ayudar a la gente a que se cure. El médico homeópata nunca ha curado a nadie. El que cura es el organismo. Nosotros somos un vehículo, un instrumento de la Naturaleza para ayudarle a que su naturaleza rectifique. Hay muchas cosas que no se tienen que curar, sino que se tienen que corregir. Diferentes hábitos: que fuma, que toma, que se desvela, que come porquerías, alimentos industrializados".

Por su parte, Lázaro, hidroterapeuta, recomienda integrar la natación en los hábitos cotidianos. Considera que "toda la gente debería practicar la natación como una forma de vida, una forma de salud".

El médico naturista, Daniel, apunta en el mismo sentido: lo importante es ayudar al paciente a cambiar de hábitos de vida. Son los pacientes más enfermos lo que están dispuestos a ello y los que le muestran el alcance de un procedimiento no convencional. Daniel admite que si la persona está muy deteriorada no se pueden hacer milagros y hay que aceptar que las expectativas de evolución están restringidas.

La biomagnetoterapeuta, Marisa, y el médico naturista, Daniel, reciben en su consulta a muchas personas con enfermedades graves, desahuciadas por la medicina científica y muy degradadas, que, según explican, mediante los tratamientos aumentan su calidad de vida y la prolongan. "Los pacientes sobre todo recuperan el ánimo, que es muy importante para curarse", explica la biomagnetoterapeuta.

Según los entrevistados, la satisfacción de los pacientes es muy alta. Los dos acupuntores resaltan el uso de productos y técnicas "naturales" que estimulan los poderes de autocuración del cuerpo y consideran que el uso de medicamentos convencionales conlleva efectos secundarios indeseados. Según el acupuntor chino, "en el cuerpo solo falta algo en el sistema inmune: falta energía" y la acupuntura contribuye a que el cuerpo se cure solo.

Uno de los homeópatas, Juan, la biomagnetoterapeuta, Marisa y el acupuntor chino, Gong afirman que pueden curar incluso enfermedades graves como el cáncer: "Todo lo que es curable se cura con homeopatía", afirma Juan.

- Las emociones y el proceso de salud-enfermedad

Los y las entrevistadas consideran que las emociones intervienen en los procesos de saludenfermedad y que están implicadas en padecimientos de enfermedades graves como el cáncer. Por tanto tratan al paciente en todas sus dimensiones: cuerpo, mente y emociones. 
La comunicación y la observación son factores clave para establecer un diagnóstico. "A veces el paciente solo quiere que le escuches. Saca lo que tiene dentro y a lo mejor ya no necesita medicamento", explica Martín, homeópata. Juan considera que el médico debe tener un conocimiento completo del paciente: "Necesitamos saber no solamente su nombre, sino sus antecedentes personales y familiares; cómo era su padre o su madre, no solamente en las enfermedades, sino en su forma de ser, de dónde ha heredado una serie de patologías o de conductas. Para nosotros es muy importante que la gente sepa que se le está atendiendo, se le está viendo a los ojos, se le está haciendo una exploración al corazón. Se le toma el pulso, se le ve la temperatura, la semblanza, la disposición de curarse. Todo lo que es el ser humano integral, en lo más profundo de su ser, de su ánimo, o sea, de su alma. Porque nosotros sabemos que solamente cuando el cuerpo y el ánimo, o sea la fuerza vital, está en equilibrio [...] el espíritu actúa en nosotros y nosotros hacemos algo trascendente".

- La satisfacción por su trabajo y la congruencia del terapeuta

Si algún aspecto destaca de las declaraciones de los terapeutas entrevistados es la satisfacción que obtienen con su trabajo y su implicación en el proceso de curación de sus pacientes. Daniel, médico y terapeuta naturista, explica que cuando descubrió los recursos alternativos sintió que se abrió "una puerta maravillosa de posibilidades impresionantes. Modificó mi condición como ser humano en muchos aspectos. Además me di cuenta que el médico debería experimentar bienestar y salud para poder hablar de ella. Antes pensaba que solo deberíamos saber anatomía y fisiología para conocer la enfermedad, pero hay que vivenciarlo", subraya. Lázaro, hidroterapeuta, afirma que "le apasiona ayudar a los pacientes. "Toda la gente viene como mínimo sus cuatro sesiones y se queda otras cuatro", añade.

Ramón, quiropráctico, se siente feliz porque no solo ayuda a los pacientes "en el plano físico", le ayuda también a cambiar la alimentación, su estado de conciencia, su estado de felicidad familiar y social. Entonces, haces cambios en individuos. Es muy difícil cambiar el mundo hay que empezar individuo a individuo".

\section{- $\quad$ Las creencias de los terapeutas}

Seis de los terapeutas manifiestan expresamente sostener creencias en la existencia de una energía vital en el ser humano, entendida como concepto de carácter esotérico utilizado en el lenguaje new age. Marisa, biomagnetoterapeuta, explica que utiliza el cuerpo de otra persona como "antena energética" para curar a un individuo que se encuentra, por ejemplo, en otro continente. Del mismo modo, José Luis, terapeuta naturista, afirma que puede lograr que el dolor del paciente pase a su cuerpo y de esa forma "disminuya sensiblemente el dolor que tiene" mediante la transmisión de energía. De igual forma, considera que puede pasar su energía al cuerpo de otra persona provocando un avance en su recuperación.

También los dos acupuntores "sacan la energía" del usuario para que él mismo pueda curarse. Otras terapias como la "biofísica aplicada a la medicina cuántica" utilizada por Abraham, médico cuántico, actúan sobre el cuerpo energético del paciente. "En la medicina cuántica se aprovecha el uso de sonidos y vibraciones de frecuencias específicas para llevar a un equilibrio el órgano dañado y eliminar el proceso mórbido que alterna el organismo", explica Abraham. Anabel, especialista en medicina natural, trata a sus pacientes mediante la medicina energética y reiki, y Daniel, médico especialista en medicina natural, utiliza la iridiología y el biomagnetismo en sus tratamientos. 
- Formación de los terapeutas

En el Cuadro I se puede ver la formación de los y las entrevistadas. De los 12 entrevistados, 11 tienen formación universitaria y 9 en el área de las ciencias de la salud. Todos los entrevistados consideran que su formación debe ser continua para poder incorporar nuevas mejoras a sus servicios. "[La homeopatía] es una medicina verdaderamente apasionante, no terminamos de estudiar nunca. Hay que estar actualizándose siempre. En la medicina no puede uno nunca quedarse estancado", explica Juan, homeópata.

- La regulación de la terapias alternativas en México

Diez de los entrevistados se muestran preocupados por la falta de regulación de la formación en terapias complementarias. "Hay diplomados y cursos en diferentes instituciones, no hay una regulación de quien los imparte. Eso es algo grave ya que no hay un respaldo de qué te enseñan y de quién lo enseña", explica Anabel, terapeuta naturista.

Felisa, acupuntora afirma lo siguiente: "Supe de un programa donde solamente fueron tres meses, los sábados, a estudiar acupuntura y a esas personas les están dejando ejercer. Hay muchas quejas de pacientes en mis consultas de daños causados por malas prácticas. Me enviaron dos practicantes de una universidad a mi consultorio y los tuve que devolver porque no tenían ni la más mínima idea de lo que estaban haciendo".

- Aplicación de las terapias complementarias en centros sanitarios

Los entrevistados coinciden en que sus tratamientos beneficiarían a muchas personas si se aplicaran en los centros sanitarios. En concreto, evitarían los efectos secundarios de los medicamentos y el alto gasto económico que conllevan. La especialista en biomagnetismo considera paradójico que solo se les permita tratar a pacientes terminales y desahuciados en las "clínicas del dolor" de los servicios de salud.

- Asociacionismo y terapias alternativas

Con la excepción de uno de los terapeutas, Juan, que forma parte de la Liga Internacional Médico-homeopática, el resto de los terapeutas no se muestran activos en un movimiento asociativo de su especialidad y son escépticos sobre la eficacia de las asociaciones que operan actualmente. En general consideran que deberían contar con asociaciones que representaran sus intereses y sirvieran como plataforma para la formación. Si bien la Universidad de Chapingo cuenta con varias agrupaciones de terapeutas, la mayor parte de los entrevistados tienen conocimiento de que existen pero no pertenecen a ninguna de ellas.

Destaca la Escuela de Osteopatía de Madrid, una organización pionera en México formando fisioterapeutas en esta especialidad, y dos asociaciones que representan a los biomagnetoterapeutas. El médico cuántico forma parte de un grupo que está en vías de constituir una asociación de especialistas en esta terapia.

\section{Conclusiones}

Diez de los doce terapeutas entrevistados son conscientes de las diferencias sociales y económicas existentes en la sociedad mexicana $y$, aunque esperan recibir una remuneración por su trabajo, son flexibles en la cuantía de sus honorarios, que se adaptan a cada paciente, y manifiestan un deseo de ayudar a los desfavorecidos. Su remuneración económica es, además, inferior a la de una consulta de medicina familiar (700 pesos) y de un especialista (más de 800 pesos). Hay que considerar que el salario mínimo en México es, aproximadamente, 88 pesos diarios. 
Entre las principales conclusiones de este trabajo hay que destacar el alto grado de compromiso de los terapeutas y de los médicos entrevistados con sus pacientes y con su trabajo. La formación de algunos de ellos es universitaria y en todos los casos complementada por cursos en distintas especialidades de la medicina complementaria.

En sus tratamientos incluyen la modificación de hábitos de vida del paciente promoviendo una dieta equilibrada, la práctica de ejercicio físico y el no consumo de tabaco y alcohol. Asimismo, alivian los estados emocionales de estrés y ansiedad por los que acuden a ellos los usuarios. Se observa una total congruencia de sus hábitos de vida con la educación para la salud que promueven ya que otorgan importancia al hecho de no fumar y beber alcohol, practicar ejercicio físico de forma regular y alimentarse de manera sana.

En cuanto a la capacidad de adaptación y de flexibilidad de los terapeutas, se constata que no solo está presente en los honorarios que cobran, sino en la firme convicción de que no existen enfermedades sino enfermos. Por ello, el pilar de su diagnóstico parte de la comunicación y de la observación. Esta implicación emocional humaniza las terapias que se aplican y en cierta manera le da sentido a su trabajo y a sus propias vidas. De este modo, la atención es personalizada y el paciente es considerado en todas sus dimensiones. Entre los terapeutas entrevistados, comprobamos que su concepción de la salud es coincidente con la de la OMS en cuanto a que consideran que constituye "un estado de completo bienestar físico, mental y social" en el que interviene el estado emocional del individuo, y en este sentido puede contribuir a la salud, al bienestar y a una atención centrada en las personas.

El modelo de relación de los terapeutas con el paciente es interpretativo o deliberativo, según la clasificación de Emanuel et al. (1999), y en cualquier caso no se corresponde con el paternalista que ha caracterizado tradicionalmente a la medicina convencional. El terapeuta considera la comunicación con el paciente un elemento esencial para lograr tener éxito con sus tratamientos.

Al carecer de una norma institucional, académica, legal y asociativa, el terapeuta se siente con la libertad de aplicar distintos remedios o terapias (Corell, 2013; Nieto-Galán, 2011) y propicia la medicina doméstica (Haro, 2000; Zolla et al., 1995), un aspecto que responde a las recomendaciones de la OMS. Fomentan así la autonomía del usuario y la responsabilidad en su proceso curativo, aspectos que la OMS destaca en la educación para la salud de la ciudadanía (OMS, 2018), con la recomendación de hábitos de vida saludables (dieta y ejercicio físico) y consumo de productos de la medicina natural que implican un menor coste.

Dicho esto, el análisis del discurso de los entrevistados ofrece algunos datos que contravienen las recomendaciones de la OMS en cuanto a la seguridad y uso racional de las terapias complementarias. En primer lugar, hay que decir que la creciente comercialización de suplementos nutricionales y vitaminas bajo el calificativo de "natural", como sinónimo de inofensivo, puede confundir a los usuarios y conllevar intoxicaciones debidas a la incorrecta administración del producto o a su interacción con otros. La misma OMS advierte sobre esta consideración (Lewis, 2002; MSPSI, 2011; OMS, 2013).

Asimismo, hay que resaltar que algunos de los participantes en el estudio consideran que pueden curar cualquier patología. Contravienen así la norma sanitaria mexicana y las 
recomendaciones de la OMS que advierten de que estos servicios deben ofrecerse únicamente como un paliativo del dolor y promotor del bienestar para asegurar el uso seguro y racional de estas prácticas y de sus productos.

Si bien no cuestionamos la recomendación de la OMS en cuanto a que las medicinas o terapias complementarias pueden contribuir a la atención sanitaria en países como México, consideramos que su utilización plantea un debate que es necesario abordar en relación a la seguridad y eficacia, así como profundizar en su reglamentación e investigación.

Aunque los terapeutas entrevistados afirman tener una amplia formación en sus áreas de trabajo, denuncian que practicantes sin formación sanitaria previa están aplicando terapias tras recibir cursos y diplomaturas no oficiales. Se calcula que existen 11.000 practicantes de acupuntura en México de los que el $60 \%$ no son médicos. Coincidimos con Torres-Vaca (2007) en que las terapias complementarias deberían regularse en México ya que se están convirtiendo en un problema de salud pública por su uso creciente y el desconocimiento de las autoridades sanitarias de la repercusión que están tenido en los procesos saludenfermedad.

Igualmente debería considerarse la eficacia de terapias que todavía carecen de fundamento científico y se basan, entre otras, en creencias alternativas sobre la energía (Barak, 2007). Afirmaciones, concretamente de la especialista en biomagnetismo, en cuanto al uso de cuerpos humanos como "antena energética" como medio para la curación, carecen de fundamento desde la ciencia. Del mismo modo, las concepciones de homeópatas y acupuntores sobre la capacidad de los seres humanos para curarse ellos mismos, resultan, como mínimo, discutibles y revelan los riesgos que corren los usuarios si confían en estas terapias para curar enfermedades graves como el cáncer. Los resultados de las encuestas de percepción social de la ciencia en México y otros estudios citados advierten sobre la necesidad de trabajar el pensamiento crítico y promover la cultura científica que cuenta con una baja consideración entre la población (CONACYT, 2016).

Finalmente, destacar que la incorporación de productos, profesionales y prácticas de las terapias complementarias debería ir paralelo al desarrollo del asociacionismo de estos especialistas. De esta forma se facilitaría el establecimiento de criterios formativos, estándares de eficacia y seguridad con el fin de que estas prácticas respondan de forma rigurosa a las recomendaciones de la OMS sobre medicina tradicional y complementaria. 


\section{Referencias bibliográficas}

ALONSO, Luis Enrique (1995): "El lugar de la entrevista abierta en las prácticas de la sociología cualitativa", en DELGADO, José Manuel y GUTIÉRREZ, Juan: Métodos y técnicas de investigación en ciencia sociales. Madrid, Síntensis, pp. 225-240.

BARAK, Judith et al. (2007): "Understanding of energy in biology and vitalistic conceptions". International Journal of Science Education, vol. 19, no 1, pp. 21-30. doi: http://dx.doi.org/10.1080/0950069970190102

CIFRHS- Comisión Interinstitucional para la Formación de Recursos Humanos en Salud (2015): "Criterios Esenciales para Evaluar Planes y Programas de Estudio para la Apertura de Carreras del Área de la Salud": http://enarm.salud.gob.mx/documentacion/criterios_esenciales/criterios.php [fecha de consulta: 15 de mayo de 2018]

COFEPRIS-Comisión Federal para la Protección contra Riesgos Sanitarios

(2016a): "Suplementos alimenticios": https://www.gob.mx/cofepris/acciones-yprogramas/suplementos-alimenticios-62063 [fecha de consulta: 12 de junio de 2018] (2016b): "Registros sanitarios de medicamentos": http://www.cofepris.gob.mx/AS/Paginas/Registros\%20Sanitarios/RegistroSanitarioMedic amentos.aspx [fecha de consulta: 6 de junio de 2018]

CONACYT- CONSEJO NACIONAL DE CIENCIA Y TECNOLOGÍA (2016): "Informe general de del estado de la ciencia y la innovación": http://www.siicyt.gob.mx/index.php/transparencia/informes-conacyt/informe-generaldel-estado-de-la-ciencia-tecnologia-e-innovacion/informe-general-2015/3814-informegeneral-2015/file [fecha de consulta: 12 de junio de 2018]

CORELL-DOMÉNECH, Mavi (2013). Científicos, vulgarizadores y periodistas: estudio y análisis de la divulgación de la ciencia en La llustración Española y Americana (1869-1898). Tesis doctoral, Universitat de València.

CRANO, William D., BREWES, Marilynn B. y LAC, Andrew (2014): Principles and methods of social research. Londres- Nueva York, Rotledge.

DENSCOMBE, Marthyn (2014): The good research guide: for small-scale social research projects. Reino Unido, MacGraw-Hill.

EMANUEL, Ezekiel J. y EMANUEL, Linda L. (1999): "Cuatro modelos de la relación médicopaciente", en COUCEIRO, Azucena: Bioética para clínicos. España, Editorial Triacastela, pp. 109-126.

HARO, Armando (2000): "Cuidados profanos: una dimensión ambigua en la atención de la salud", en PERDIGUERO, Enrique y COMELLES, José Ma: Medicinas y cultura. Estudios entre la medicina y la cultura. Barcelona, Ediciones Bellaterra, pp.101-162.

HERNÁNDEZ, Jethro (2017): "Homeopathy 'for Mexicans': Medical Popularisation, Commercial Endeavours, and Patients' Choice in the Mexican Medical Marketplace, 18531872". Medicina e Historia, vol. 61, nº 4, pp. 568-589. 
IDOYAGA MOLINA, Anatilde (2002): Culturas, enfermedades y medicinas. Reflexiones sobre la atención de la salud en contextos interculturales de Argentina. Buenos Aires, Prensa del Instituto Universitario Nacional del Arte.

KOOREMAN, Peter y BAARS, Enrik W. (2012): "Patients whose GP knows complementary medicine tend to have lower costs and live longer". The European Journal of Health Economics, vol. 13, no 6, pp. 769-776. doi: http://doi.org/10.1007/s10198-011-0330-2

LEWIS, Ricki (2002): "Dietary Supplements", en SHERMER, Michael (Ed.): The skeptic Encycolpedia of Pseudoscience. Vol. 1, 2, 3. EE. UU., California: Skeptics Society, Skeptic magazine, pp. 85-92.

MARSHALL, Catherine y ROSSMAN, Gretchen. B. (2014): Designing qualitative research. Londres, Sage publications.

MENÉNDEZ, Eduardo L. (1994): "La enfermedad y la curación. ¿Qué es medicina tradicional?". Revista Alteridades, vol. 4, no. 7, pp. 71-83.

MENÉNDEZ, Eduardo L. (2002): "El punto de vista del actor: homogeneidad, diferencia e historicidad", en MENÉNDEZ, Eduardo L.: La parte negada de la cultura. Relativismo, diferencias y racismo. Barcelona, Editorial Belaterra, pp. 309-374.

MSPSI- Ministerio de Sanidad, Política Social e Igualdad (2011): Análisis de la situación de las terapias naturales. Madrid: Ministerio de Sanidad, Política Social e Igualdad. http://www.mspsi.gob.es/novedades/docs/analisisSituacionTNatu.pdf [fecha de consulta: 6 de junio de 2017]

MOLINA-RODRÍGUEZ, Juan Francisco et al. (2006): Utilización de servicios de salud en México. Salud en Tabasco, vol. 12, nº 1, 427-432.

NIETO-GALÁN, Agustí (2011): Los públicos de la ciencia. Madrid, Ambos Mundos.

OCED- Organización para la Cooperación y el Desarrollo Económicos (2017): "Health at a glance. OECD Indicators": https://www.oecd.org/mexico/Health-at-a-Glance-2017-KeyFindings-MEXICO-in-Spanish.pdf [fecha de consulta: 6 de junio de 2018]

OMS-Organización Mundial de la Salud:

(2013): Estrategia de la OMS sobre medicina tradicional: http://apps.who.int/medicinedocs/documents/s21201es/s21201es.pdf [fecha de consulta: 5 de junio de 2018]

(2018). México. Resumen: estrategia de cooperación. http://apps.who.int/iris/bitstream/handle/10665/250865/ccsbrief_mex_es.pdf?sequence= 1 [fecha de consulta: 5 de junio de 2018]

PASARÍN, Lorena (2011): "Itinerarios terapéuticos y redes sociales: actores y elementos que direccionan los procesos de salud/enfermedad/atención", en Los aportes del Análisis de Redes Sociales a la Psicología. Mendoza, Editorial de la Universidad del Aconcagua. 
PL-Parlamento Latinoamericano (2009): "Anteproyecto para la ley marco en materia de medicinas complementarias":

https://www.gob.mx/cms/uploads/attachment/file/37974/LeyMarcoMedicinasCompleme ntarias.pdf [fecha de consulta: 12 de junio de 2018]

SSGM-Secretaría de Salud del Gobierno mexicano:

(2012): "NORMA Oficial Mexicana NOM-017-SSA3-2012, Regulación de servicios de salud. Para la práctica de la acupuntura humana y métodos relacionados": https://www.gob.mx/cms/uploads/attachment/file/37973/NOMo17.pdf [2018-6-12]

(2018a): "Medicinas complementarias": https://www.gob.mx/salud/acciones-yprogramas/medicinas-complementarias?state=published [fecha de consulta: 6 de junio de 2018]

(2018b): "Modelos clínico-terapéuticos y de fortalecimiento de la salud": https://www.gob.mx/cms/uploads/attachment/file/37989/FortOtrosModelosMedicos.pdf [fecha de consulta: 6 de junio de 2018]

TORRES-VACA, Marisela (2007): "Prevalencia en el uso y aceptación de terapias alternativas en alumnos de tres planteles de la Universidad Nacional Autónoma de México". Vertientes. Revista Especializada en Ciencias de la Salud, vol. 10, n 1-2, pp. 1216.

YOW, Valerie Raleigh (2014): Recording oral history: A guide for the humanities and social sciences. Londres, Rowman \& Littlefield.

ZOLLA, Carlos y MELLADO, Virginia (1995): "La Función de la Medicina Domestica en el Medio Rural Mexicano", en GONZÁLEZ-MONTES, Soledad: Las Mujeres y la Salud. México, D.F., El Colegio de México, pp. 71-92. 